\title{
Beiträge zur Kenntniss der pathogenen Gregarinen.
}

IV.

Gregarinenformen innerhalb der Blutzellen bei Schildkröten, Eidechsen, Vögeln und von Malariakranken. ${ }^{1}$

$$
\text { Von }
$$

Dr. L. Pfeiffer

in Weimar.

Von Professor Dr. Danilewsky in Charkoff sind jüngst in dem Blute von Schildkröten, Eidechsen, Fischen und Vögeln Schmarotzerformen aufgefunden worden, die denen aus Malariablut sehr nahe stehen. Abgesehen von der dadurch erhaltenen weiteren Bestätigung, dass parasitäre Vorgänge grober Art im rollenden Blute selbst sich abspielen können, wird durch die Infectionsvorgänge inmerhalb der Blutzellen eine ganz neue und weite Perspective für die Erkenntniss der biologischen Vorgänge, der histogenetischen Bedeutung-des Zellkernes u. s. w. eröffinet.

Soweit zunächst Gregarinenformen bei Emys lutaria, Testudo campanulata, Lacerta viridis und Vögeln in Frage kommen, kann Verfasser die von R. Danilewsky geschilderten Vorkommnisse aus eigener Anschauung bestätigen und kaum etwas Neues hinzufügen. Für Malaria hatte Verfasser bei drei in Deutschland entstandenen Erkrankungställen negatives, bei einem aus Italien kommenden Kaufmann aber positives Resultat zum Vergleich. Letzterer hatte in jedem 90. bis 100. rothen Blutkörperchen je eines der charakteristischen sichelförmigen Körperchen. Amöboide oder Plasmodienformen bei den Malariakranken hat Verfasser nicht finden können, wohl weil alle Kranken bereits mit Chinin behandelt worden waren.

Die von R. Danilewsky im Blute der Vögel beobachteten amöboiden oder Flagellatenformen, von ihm zu Polimitus malariae gerechnet, kamen im Sommer 1889 bei 40 lebenden Vögeln nur in einer Schleiereule vor.

1 Das Manuscript wurde im December 1889 abgeschlossen. Seitdem sind erschienen der Aufsatz ron Plehn in Bd. VIII, Hft. 1 dieser Zeitschrift und von R. Paltauf in der Wiener klinischen Wochenschrift. 1890. Nr. 2 u. 3. 
Es wurden, entsprechend den Mittheilungen von Danilewsky, nur Nesthocker (Würger, Mantelkrähe, Elster, Eule, Bussard), aus Thüringen, Franken, Hannover, ausgewählt. Es müssen auch hier endemische Einflüsse sich geltend machen, insofern nach Danilewsky das Vorkommen des Polimitus arium in Charkoff und im Jardin des plantes zu Paris ein gewöhnliches ist.

Um Missverständnissen vorzubeugen, sei ausdrücklich betont, dass hier nur das Vorkommen von Gregarinenformen innerhalb der Blutzellen beschrieben werden soll und dass die Beziehungen der Plasmodien zur Malariainfection nicht Gegenstand der nachfolgenden Mittheilungen ist.

\section{Haemogregariua cistudinis s. Stepanowi (R. Da nilewsky).}

(Fig. 1 bis 5.)

Es handelt sich hier um eine Gregarine, speciell um eine Eimeria ${ }^{1}$ oder um eine Sporidienform, welche ihren Entwickelungsgang ganz innerhalb der rothen Blutkörperchen von Emys lutaria, auch ron Testudo campanulata ${ }^{2}$ durchläuft und im Vermehrungszustand die Hülle der Blutzelle als Cystenhülle verwendet. Die Infection mit 1, 2 und selbst 3 Parasiten
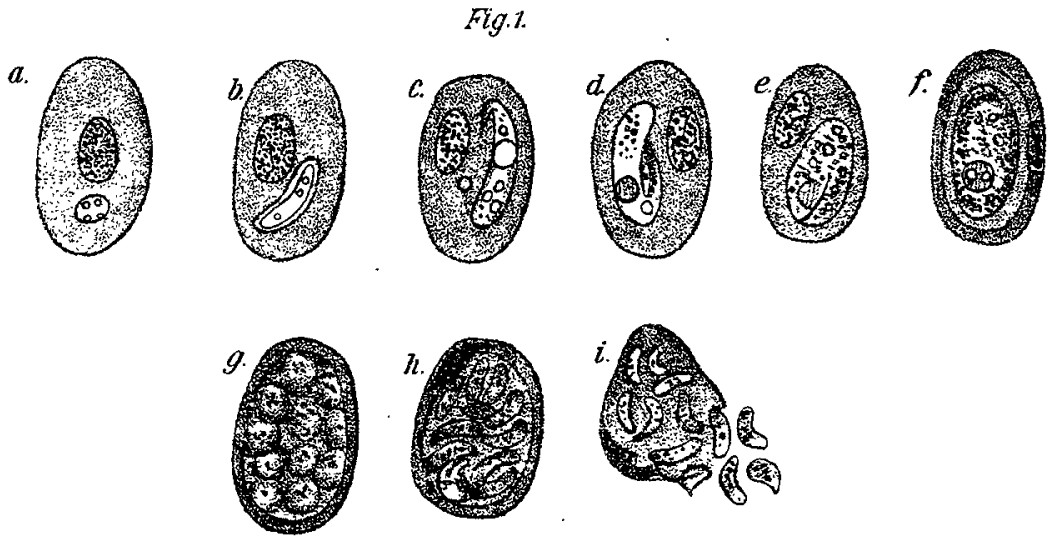

Entwickelung der Haemogregarina cistudinis. Vergrösserung 1500/1. Nach frischen Blutpräparaten. $a$ bis $e$ Vegetatives Stadium. $f$ bis $i$ Vermehrung.

findet im Hämatoblastenstadium (oder noch früher?) statt; die Cystenbildung an ruhigen Stellen des Kreislaufes, z. B. im Knochenmark, in der Niere, mit neuer Entstehung und Ausstreuung ron 8, 16 oder 24 sichelförmigen Keimen. Der Parasit hat zu allen Zeiten einen deutlichen centralen Kern, keine Vacuolen, ständig einige bis riele Gregarinenkörner

1 Nach Verfassers Auffassung.

2 Bezogen von Ge u pel-Leipzig. 
nach den Enden zu. Amüboidzustände kommen nicht vor. Die frisch ausgeschlüpften sichelförmigen Keime sind im Blutplasma beweglich; ebenso alte exkapsulirte Individuen. Innerhalb der Blutzelle ist die Hämogregarina cistudinis unbeweglich.

Der Parasit ist zuerst von Professor R. Danilewsky in Emys lutaria gefunden worden und kommt vor in der Umgegend von Charkoff, Odessa, in Südsibirien. Verfasser hat denselben nach mehrfach vergeblichem Suchen, aus Emys Iutaria, von Geyer in Regensburg bezogen, beobachtet; der Fangort der Schildkröten ist nicht ermittelt. Auf je 10 Exemplare waren $1-2$ inficirt, sowohl junge als alte; einige alte hatten auf 10 Blutkörperchen des Schenkelhautblutes je ein inficirtes, und im Knochenmark noch mehr. Die von Danilewsky ungeführten Thatsachen kaun Verfasser durch eigene Zeichnungen bestätigen.

Schenkelhautblut. In Präparaten mit 0.6 procentigem Salzwasser oder mit Kammerwasser des Schafauges erscheint der Parasit bei 300 facher Vergrüsserung als deutlich heller Fleck innerhalb der rothen Blutscheibe, zur Seite des Blutzellkernes gelagert. Die relativ häufig vorkommenden grossen Formen haben anscheinend die Länge des grösseren Blutkörperchendurchmessers, sind aber doppelt so lang, da zwei Hälften in Taschenmesserform mittelst eines Knickes dicht aneinander gelagert sind (Fig. $1 d$, $1 e, 5 a, 5 b, 4 d, 3 a, 2 a)$. Uebergangsformen, von einfachen kurzen Stäbchen zur Sichelform, weiter nit Umbiegung des einen dünneren Endes bis zu solchen Taschenmesserformen, sind stets vorhanden (Fig. $1 a-e)$. Ein Zusatz von Kammerwasser, mit etwas Methylenblaupulver versetzt, färbt zunächst den Zellkern der Blutscheibe, dann das Zooid und auch den Parasiten. In den auf gewöhnliche Weise hergestellten Trockenpräparaten färben sich Kern, Kernkörperchen und Granulationen des Parasiten schön roth mit einer Lösung ron Carmin in schwacher Osmiumsäurelösung (Danilewsky). Zur Doppelfärbung eiguet sich HämatoxylinEosinlösung; der Parasit erscheint roth neben dem dunkeln Blutzellkern, besonders nach vorausgegangener kurzer Extraction des Hämaglobulins mittelst schwacher Bor- oder Essigsäure (2 Procent). (Pfeiffer).

Zur weiteren Orientirung mögen folgende Maasse dienen für Emys lutaria, welche mit denen von Danilewsky übereinstimmen:

Reife Blutzellen . . . 0.0216-0.027 lang -0.011-0.0114 breit, Kern derselben . . $0.004-0.007$ "

Wikrocyten. . . . . $0.0126-0.0180 "$

Kleinste Hämatoblasten

Reifer Parasit, geknickt

" " gestreclit

Jüngste sichtbare Form

in den Mikroeyten. .

0.007 .

0.028

$0.042 "$

$0.004-0.005$. 
Die jüngste intracelluläre Form (Figr. $1 a, 4 a$ ) erscheint als helles Bläschen, rund oder oval neben dem Blutzellkern; besonders deutlich nach Zusatz von etwas Methylenblaulösung (in Kammerwasser, Imnionwasser, Hydrocelenflüssigkeit u. s. w.). Besonders die birnenfürmigen oder mondsichelförmigen Hämatoblasten beherbergen diese jüngste Form.

Die nächste Form, in der Grösse des Blutzellenkernes, erscheint in den Mikrocyten, in den Hämatoblasten und auch in den rothen Blutzellen als heller Fleck, aber mit einigen Granulationen und mit etwas mehr verwaschenem Umriss (Fig. 1a). Mit Verschärfung der Contour wird daraus ein rundes oder längliches wurmartiges Kürperchen, mit einigen runden, glänzenden Granulationen an beiden Enden und mit hellem rundem Kernfleck in der Hitte (Fig. 1b). Die Farbe ist bläulich weissglänzend und bleibt so durch den ganzen Lebenslauf hindurch. Bei noch weiterem Wachsthum biegt sich eine Art Schwanz um. zur schou

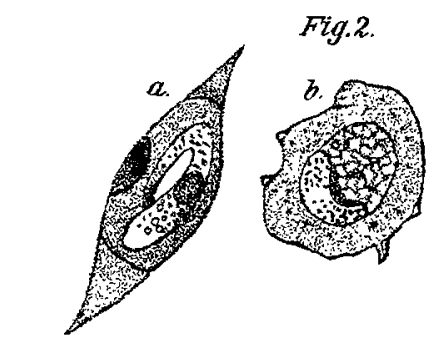

Verḡösserung 1500/1. Lebendes Material. a Inficirter Hämatoblast, an den Enden blutbraun gefärbt und ausnahmsweise mit einer bereits ausgewachsenen Hämogregarine besetzt. $b$ Halbwüchsiger Parasit in einem amöboiden Leukocyter.
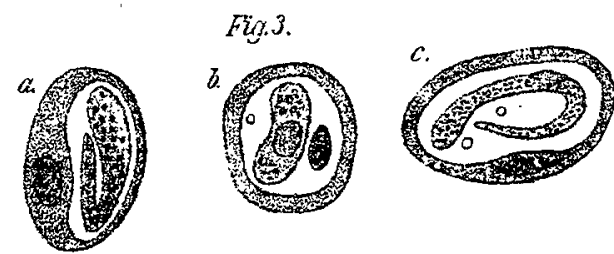

Vergrösserung 1500/1. Hämocyten mit brauner Randzone. Kern bald ausserhalb ( $a$ und $c$ ), bald innerhalb $(b)$ einer Scheinkapsel gelagert.

beschriebenen Taschenmesserform den Uebergang bildend (Fig. 1c, 1d). Ein Ecto- und Entoplasma ist nicht differenzirt, doch scheint, nach spïter zu beschreibenden Bewegungsvorgängen, das Centrum von flüssiger Consistenz Vacuolen sind nur an absterbenden Parasiten zu beobachten (Fig. $5 g$ ). Der Kern, mit einigen Kernkörperchen, ist ron weicher Beschaffenheit, er nimmt Antheil an den ringtörmig sich folgenden Wülsten, wenn der Parasit sich im Blutserum fortbewegt (Fig. 5f). Die Granulationen (4-6) an beiden Enden bestehen aus Gregarinenfett.

Die Gestalt der inficirten Blutzelle wird durch kleine oder grosse Parasiten nicht verändert; das gilt für die erst halbgefärbten Hämatoblasten ebenfalls (Fig. 2a). Dass bei Emys die Parasiten auch in Leukocyten eindringen, dafür spricht Fig. 26 (phagocytärer Process?). Auch die Färbung der rothen Blutkörperchen bleibt gut erhalten; gewöhnlich 
ist der Parasit von hämoglobinhaltigem Plasma, dem Zooid, dicht umHossen. Doch treten auch Formen auf mit einem schmalen hellen Saum zwischen der gefärbten Randpartie der Blutzelle und dem Parasiten. Der Kern der Blutzelle liegt dabei bald innerhalb (Fig. $3 b$ ), bald ausserhalb (Fig. $3 a, c$ ) des Ringes und ist auch mit Reagentien eine Kapsel um den Parasiten im Zooid nicht nachweisbar. Freigewordene grössere Parasiten schleppen zuweilen eine Kapsel mit sich herum, wie eine Schnecke ihr allerdings besser erhaltenes Gehäuse; aber diese Kapsel ist meist faltig und geschrumpft, beutelartig und ist als die Hülle der zu Grunde gegangenen Wirthszelle zu betrachten (Fig. 5 $a, b, c)$. - In den Blutzellen mit grossem Fremdling ist manchmal nur ein ganz schmaler Randring ron Blutfarbe erhalten; ganz entfärbte sind selten. Pigmentzörner im Zooid kommen nicht vor.

Der Kern in den inficirten Blutzellen ron Emys lutaria erleidet auffallend wenig Veränderungen; sehr selten ist seine Contour gestört. Eine Einkerbung, ein Zerfall, wie dies bei Lacerta die Regel ist, kommt kaum vor, selbst wenn er durch einen grossen Fremdling ganz an die Zellwand angedrückt wird.
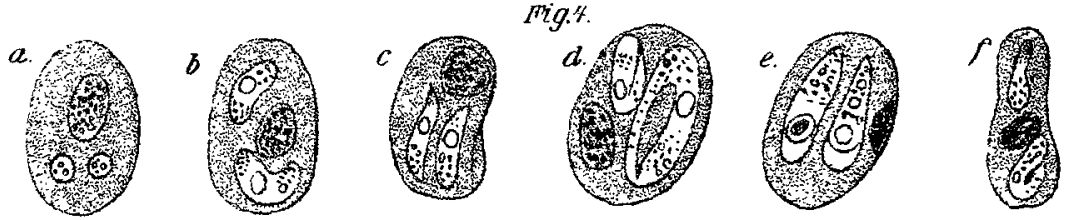

Vergrösserung 1500/1. Zwillingssinfection von Hämocyten. In Fig. $4 d$ ist ein Grössenuntersehied der beiden Fremalinge ausnahmsweise vorhanden.

Infectionen von Hämatoblasten oder Bluthörperchen mit 1, 2 und selbst 3 Parasiten sind nicht selten, besonders im Knochenmark (Fig. 4). Zwillinge sind meist gleich gross und also wohl auch meist gleich alt. Bei ungleicher Grösse hönnte man, wie dies in den Epithelzellen der Niere von Helix arbustorum, hortensis, frutidum, Succinea Pfeiferi und putris durch eine Coccidie (Ǩlossia Soror Aimé Schneider) rorkommt, an eine Successivinfection oder Superföcundation denken (Fig. $4 d$ ). Direct beobachtet ist das Eindringen der Fremdlinge in die Blutzellen noch nicht. In jungen Schildkröten sind meist nur kleinere Formen und diese zahlreich in den Hämatoblasten vertreten; bei älteren Schildkröten finden sich in den rothen Blutzellen mehr die ausgewachsenen Parasiten. Es wird somit das Wachsthum der Blutzellen durch die Anwesenheit von Parasiten nur wenig gestört. Da bei ganz alten Schildkröten und im Winter die Vermehrung der Parasiten einen Stillstand macht, nimmt Danilewsky mit Recht an, dass die Entwickelung des Parasiten inner- 
halb der lebensfähig bleibenden Bluthörperchen sich auf Monate erstreckt - eine Langlebigkeit, die sich ganz gleich bei den Sarcosporidien findet.

Frei zwischen den Blutzellen sich findende sichelförmige Körperchen benennt Danilewsky als exkapsulirte Form (Fig. 5). Sie sind zum Theil beweglich und ihre Geburt aus den Blutzellen lässt sich unter dem mit Wachsfüsschen und mit Fettrand geschützten Deckglas beobachten, sobald Abkühlung (und Sauerstoffmangel!) eingetreten ist. Sie erscheinen zunächst noch an den Enden gleichsam zusammengeknotet, 0.028 lang (Fig. 5e), haben aber die Neigung, sich zu strecken, indem sie sich von der anhaftenden, wie ein loser zerknitterter Beutel erscheinenden Ze hülle, abtrennen (Fig. $5 a, b$ ). Die mittlere Länge ist, wenn gestreckt,

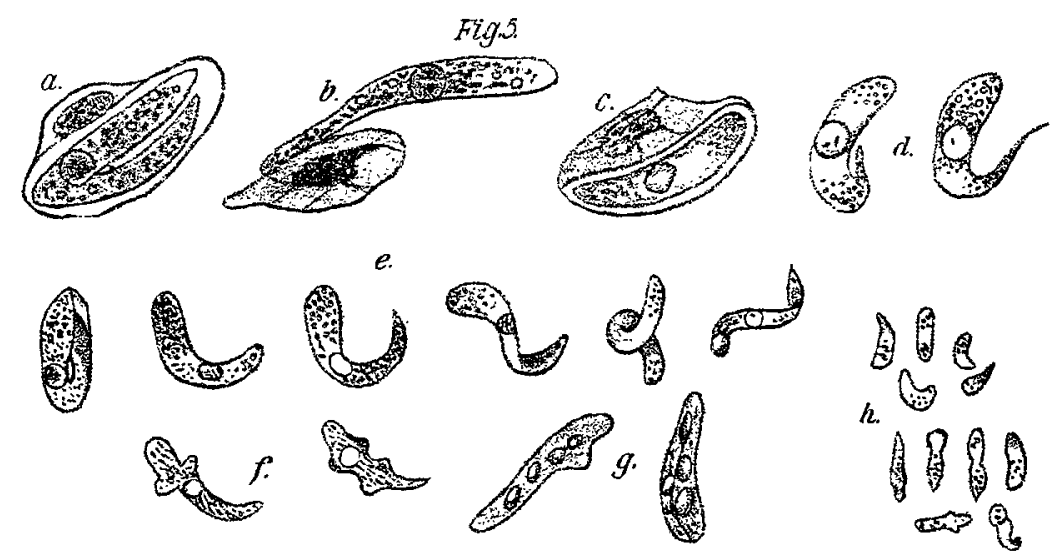

Die Bewegungen der Haemogregarinae cistudinis. Vergrösserung 1500/1. a bis $f$ der erwachsenen, $h$ der ganz jungen Formen. $a, b, c$ sind entfärbte und gefaltete Hämocyten. $e$ sind sechs aufeinander folgende Bewegungsformen eines eben frei gewordenen Parasiten um eine feststehende Axe. $f$ Die geradlinige Fortbewegung. $g$ Abgestorbenes Exemplar. $h$ Keime mit Bewegung.

das Doppelte der Blutzellenlänge: 0.03 bis $0.04 \mathrm{~mm}$. Der Körper dieses Parasiten ist, wie seine Bewegung lehrt, cylindrisch, an einem Ende stumpf, am anderen spitzer abgerundet. Die Structur ist homogen, Ectound Entosark sind nicht zu unterscheiden, ebensowenig ein Sarcocyt. Die Farbe ist bläulich, manchmal bei directer Beleuchtung mit einigen dunkleren Querringen. Der Kern liegt in der Mitte, hat scharfen runden oder ovalen Rand und einige dunklere Körnchen (Fig. $4 a-b$ ).

Die an diesen exkapsulirten Parasiten zu beobachtenden Bewegungsvorgänge sind doppelter Art, immer aber sehr träge. Die Fortbewegung geschieht, immer vorwärts, durch quere Wulstungen, in 3 bis 4 Reihen sich folgend und den Kern mit einschnürend (Fig. $5 f$ ). Ausserdem finden, 
ganz so wie bei Klossia, Eimeria, bei Sarcosporidien - noch Beugungen bis fast zur Schleifenform, Streckung und auch Schraubendrehung mit Rückstreckung statt (Fig. ̃o). Mit der Bewegung von Englena kann diese langsame Drehung um eine feststehende Axe nicht verglichen werden. Sie wird bei Lacerta innerhalb des Blutkörperchens normaler Weise aufgeführt in ganz überraschender Weise. Involutionsformen kommen vor; ebenso Monstrositäten. Die unter dem Deckglas nach ca. 10 Minuten sich exkapsulirenden Formen werlen bald vacuolis und gehen raseh zu Grunde (Fig. 5g). Besonders das Blut junger oder hungernder Schildkröten hat viele exkapsulirte Exemplare, darunter anch viele halbwüchsige.

Knochenmarkblut. Dasselbe ist ausgezeichnet durch weitere Entwickelungsstadien der ausgewachsenen Parasiten, sowie durch das Vorkommen zahlreicher ganz kleiner, freier Formen (Fig. 5h). Das Nierenblut verhält sich ähnlich, während die. Milz bei Emys keine Sonderstellung einnimmt. Zur Cntersuchung eignet sich besonders das aus dem rothen Kinochenmark der Epiphysen ausgequetschte Blut.

Die interessanteste und für den Entwickelungsgang ausschlaggebende Formenreihe ist die in Fig. $1 f, g, h, i$ abgebildete, welche aus ganz jungen Schildkröten erhalten werden kann und welche ganz an eine sporenbildende Cyste einer Coccidie, speciell an die einsporige Eimeria oder noch mehr an eine Sporidiencyste oder an Synchytrium erinnert, wobei die Hülle des befallenen Wirthes als Cystenwand mit Verwendung gefunden hat. Die Blutzellen mit Theilungsvorgängen im Parasiten sind meist etwas grösser $(0.025$ bis $0.03 \mathrm{lang}, 0.016$ breit) als gesunde Blutzellen $(0.0216$ bis $0.027: 0.011){ }^{1}$

Aus dem reifen Parasiten wird zunächst ein ovaler, feinkörniger, hellglänzender Körper, an dem die Mittelfurche der Taschenmesserform nicht mehr zu unterscheiden ist, in dem aber der Kern deutlich blieb (Fig. 1f). Umgeben ist derselbe immer noch von einem schmalen blutgefärbten Saum am Blutzellrand, mit dem eingebetteten Zellkern. Dis nächste Stadium ist repräsentirt durch ein Blutkürperchen gleicher Beschaffenheit, dessen Fremdhörper aber 8, 16 oder 24 Kugeln (Fig. 1g) in sich enthält. Ein weiterer Entwickelungszustand zeigt diese hyalinen Kugeln etwas länglich und fein gelörnt. Schliesslich finden sich auch noch Blutkörperchen, die wie die Sporen einer Klossia, einer Eimeria oder einer Sporidie, bis auf einen schmalen braunen Randring mit kleinen sichelförmigen Keimen erfüllt ist (Fig. 1h): Auch in diesem Stadium bleibt der Blutzellenkern noch dentlich. Beim Platzen solcher Cystenzellen treten Keimlinge aus (Fig. $1 i$ und $5 h$ ), die sich morphologisch nicht

${ }^{2}$ Die Maasse nach Danilewsky's Original und verglichen. 
unterscheiden von den innerhalb der Hämatoblasten vorkommenden kleinsten Gregarinenformen; sie sind hell, haben an beiden Enden Fetttröpfchen, ohne deutlichen Kern, und bewegen sich ganz so wie die im Körperblut zu findenden, mit wurmartig fortrückenden Quereinschnürungen, neben leichter Beugung und Streckung um eine feststehende Axe.

Ebensolche sichelförmige Embryonen, 0.004 lang, sind zahlreich frei im Knochenmarkblut (Fig. $\overline{\mathbf{b}} h$ ). Amöboide Zustände liommen nicht vor, im Gegensatz zu den Sporidien und zu den im Fogelblut und im Malariablut vertretenen Formen.

Ob die Infection durch noch viel kleinere im Blute rorkommende, den Gregarinenkörnern ähnelnde Gebilde bedingt sind, wie Danilewsky anzunehmen geneigt ist, bleibt noch offene Frage. Allerdings sind die kleinsten vacuolenartigen Fremdlinge in den Hämatoblasten noch kleiner als die den Cysten entschlüpfenden sichelfürmigen Keime. Möglich, dass in den Nieren von Danilewsky beabachtete kleinste racuolenartige Gebilde daselbst als eine Art von Dauerspore sich bilden und dass es sich bei diesen Parasiten gar nicht um eine Successirinfection der Blutzellen in demselben Wirth handelt, wie wir das in Abschnitt I bei den Mikrosporidien der Pebrine beschrieben haben und wie es auch von den Sarcosporidien wahrscheinlich ist. Sprossbildnngen, die Celli und Guarnieri innerhalb der inficirten Malariablutzellen an den fremden sichelförmigen Körperchen direct beobachtet haben, kommen bei Emrs nicht vor.

In den Ausleerungen von stark inficirten Schildkröten, leider meist mit massenhaften Helminthen gemengt, hat Verfasser vergeblich nach Gregarinenformen gesucht.

Besondere Krankheitserscheinungen sind bei stark inficirten, bei jungen und alten Schildkröten, nicht aufgefallen.

\section{Haemogregarina lacertae Danilewsky.}

(Figg. 6 und 7.)

Diese Gregarinenform ist 1884 ron Danilewsky aufgefunden in Lacerta viridis und agilis in der Lmgegend ron Charkoff. Verfasser hat sie jüngst in 12 Exemplaren von Lacerta viridis, bezogen von GeyerRegensburg, ebenfalls gefunden; in einer derselben war jedes zehnte Blutkörperchen befallen. Die von Danilewsky beschriebenen Verhältnisse kamn Verfasser auch hier durch nachstehende Zeichnungen bestätigen.

Im grossen Ganzen verlüuft die Infection hier ganz ähnlich wie bei Emys, aber folgende wesentliche Enterschiede sind zu beachten. Der Parasit ist bei Lacerta nur halb so gross. insofern er sich nicht taschen- 
messerartig zusammenlegt. Die mittelgrossen Parasiten vollführen alle 1 bis 5 Minuten innerhalb der Blutzelle ein und dieselbe Bewegung aus, um den Blutzellkern herum unter Beugung, Streckung oder auch Spiraldrehung ihrer Längsaxe (Fig. 6). Die ausgewachsenen Parasiten $(0.015$

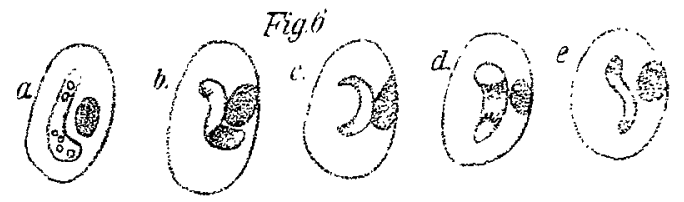

Vergrüsserung 1500/1. Bewegungsformen innerhalb noch wenig veränderter rother Blutzellen von Lacerta.

bis 0.017 lang, 0.003 bis 0.004 breit) werden sehr träge oder sistiren in der Bewegung unter gleichzeitig eingetretener Zerstörung der Wirthszelle (Fig. $7 b, c, d . f, g$ ). Das Zooid wird rollständig entfärbt, körnig; die Hülle bekommt Faltungen und der Zellkern zerbröckelt (Fig. 7g).

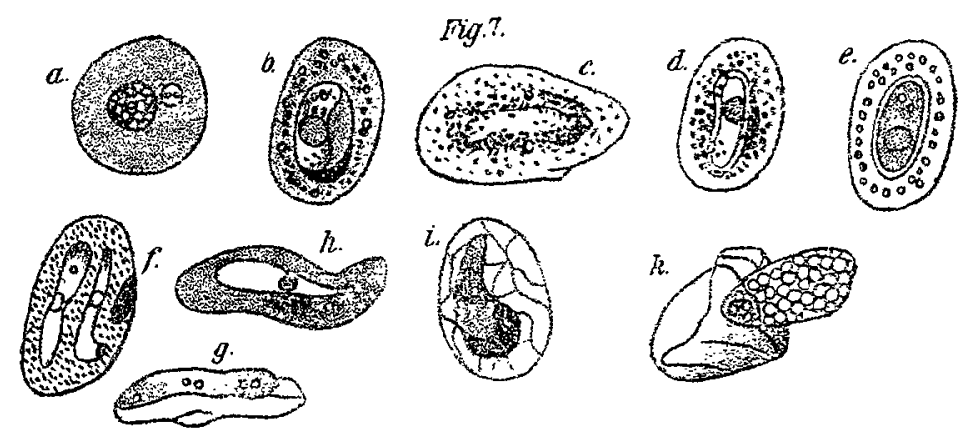

Vergrösserung $1500 / 1$. Entwickelung der Haemogregarina lacertae. $a$ bis $f$ Lebendes Naterial. $\hbar$ u. $i$ Gefärbt-mit Phloxin-Methylenblau. $e$ u. $k$ Zweifelhafte Cystenformen.

Mit Bromwasser wird eine Hüllhaut am Parasiten deutlich (Fig. $7 d$ ).

Die kleinsten Parasiten haben dasselbe Verhalten, wie soeben bei Emys geschildert worden ist; die Blutfarbe der Wirthzelle ist dabei ebenfalls noch erhalten (Fig. 7a). Bei weiterem Wachsthum wird der Wirthskern an die Zellwand gedrückt, wird breit oder auch sichelförmig und zerfällt bald in zwei und mehr Theile. Die rhythmischen Beugungen des Parasiten setzen auch den Blutzellkern in Bewegung, wobei zuweilen der Kern zwischen die sich nähernden Sichelenden gefasst wird und ein Hinderniss der Bewegung abgiebt. Dieses Spiel kommt in überraschender Weise zur Anschauung, wenn durch Zusatz ron schwach blau gefärbtem Kammerwasser zunächst der Blutzellkern etwas Farbe annimmt und der 
Parasit noch einige Bewegungen ausführt, ehe auch er gefärbt wird und abstirbt.

Die ganz jungen Formen sind schwerer aufzufinden als bei Emys; mit Hülfe der Eiweissfarblösung gelingt es auch hier, neben dem Blutzellkern einen hellen Fleck $(0.010$ bis 0.012$)$ mit ganz feinen Granulationen zu entdecken, zunächst noch ohne eigene Kernandeutung. Beim Errwärmen sind Contourveränderungen an dem Fleck zu bemerken.

Freie Formen im Blut sind luur oral oder mehr säbelförmig gestreckt, in der Länge den Blutzellen entsprechend. Sie sind durch bläulichen Glanz ausgezeichnet und bewegen sich selbstständig zwischen den Blutscheiben, ganz in der Weise, wie die sichelfürmigen Körperchen der Coccidien, der Sporidien und wie die exkapsulirten Hämogregarinen ron Emys.

Amöboidformen hat Verfasser nicht auffinden lïnnen. Frei im Blut schwimmend kommen noch kleinste, unbewegliche Formen vor, die durch ihren Glanz an Gregarinenkörner erimnern. Die Bedeutung derselben ist vollständig unklar.

Die Vermehrung der Haemogregarina lacertae ist eine andere, als die der Haemogregarina cistudinis; im Knochenmarke fehlen die für Emys so charakteristischen Cysten. Die besondere Hüllhaut im Inneren der degenerirten Blutzellen um ausgewachsene Parasiten herum, sowie ferner Formen, rorstehend in Fig. $7 e$ und $k$ abgebildet, lassen eine anders geartete Anpassung des Parasiten an die weniger widerstandsfähigen Blutzellen der Eidechsen und eine andere Vermehrung als bei Emys vermuthen.

Besondere Krankheitserscheinungen bei den Eidechsen sind nicht beobachtet. Nach längerem Zusammenleben in einem Kä̈ig hatten auch die anfangs nicht inficirten Exemplare solche Parasiten erhalten.

\section{Haematozon arium Danilewskr.}

(Figg. 8 und 9.)

Danilewsky beschreibt aus dem Blut ron rögeln. speciell nur ron Nesthockern, fünf rerschiedene Formen eines (oder mehrerer? Verfasser) Parasiten:

A. Microbes paludiques.

1. Psendorermiculi (Fig. 8).

2. Pseudovacuolae s. Cytozoa (Fig. 9),

3. Polimitus avium, (Fig. 10). 
B. Trypanosomes et Trypanomonades.

4. Trypanosoma avium,

5. Pseudospirilla avium.

Verfasser hat im Sommer und Herbst 1889 in Jitteldeutschland nach inficirten Vögeln gesucht und schliesslich in einer Schleiereule die in Fig. 8 bis 10 abgebildete Form gefunden. Es soll der erste Frühling die geeignete Zeit sein und hat Danilewsly sogar die ron den Eltern im

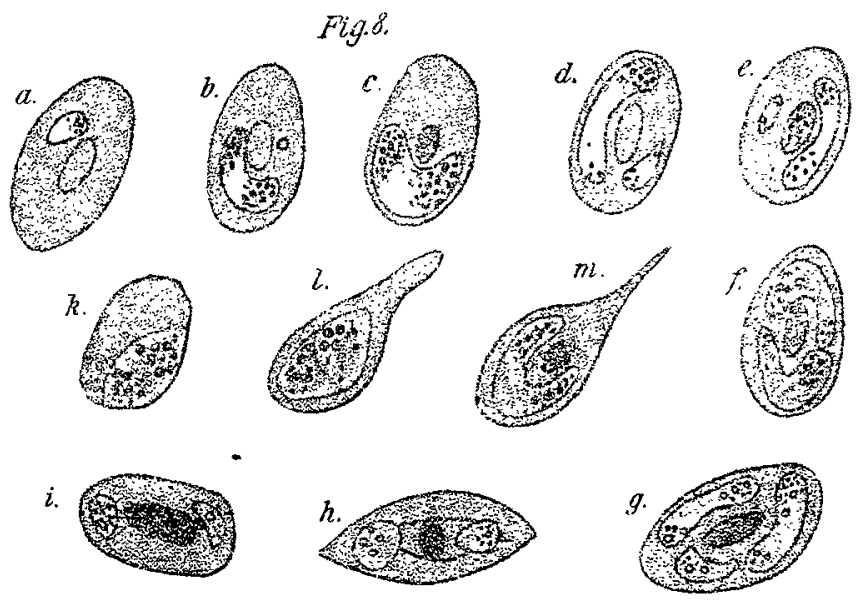

Vergrösserung 1500/1. Haematozoa avium. $a, b, c$ die gewöhnliche Form, $l$ in Hämatoblasten. $d, e, f, i, h, g$ Mehrlingsinfection. $k$ Cystenbildung (?) nach D anile ws ky.

Nest gefütterten Vögel inficirt gefunden. Polimitus mit Flagellaten und die amöboiden Formen verlangen Beobachtung des lebenden Materiales auf gewärmtem Mikroskop. Die mit sichelförmigen Körpern behafteten rothen Blutscheiben (Pseudorermiculi D.) der Tögel zeigen im Ganzen das gleiche Verhalten (Fig. 8) wie bei Emys. Die Hülle ist gut erhalten; das Hämoglobin aber ist bei grossen Parasiten zu dunklen Punkten zerfallen. Der Blutzellkern ist erhaiten (Safraninfärbung) und meist central gelegen.

Wird das Blut auf dem Objectträger warm gehalten, so bekommt (Fig. 10) das befallene Blutkörperchen nach 10 Ninuten eine seitliche Ausbuchtung, aus welcher nach weiteren 40 bis 60 Hinuten das sichelförmige Körperchen ausschlüpft, 15 bis 17 Mikrom. lang. Es ist hyalin, hat Kernfleck und ist beweglich, genau wie bei Emys und Lacerta. Solche Formen finden sich auch frei in dem eben entnommenen Vogelblut, 10 bis 19 Milrom. lang und mit deutlichen Querleisten versehen. 
Jüngere Parasitenstadien, Cytozoa und Pseudovacuolae nach Danilewsky, erscheinen als heller bläschenartiger Fleck im Innern der Hämocyten der Vögel (Fig. Sa) ganz wie bei Emys, Lacerta und von dem Blutzellkern durch Farbstoffe, durch Säuren, Brom- und Chloroformwasser leicht zu differenziren. In Hämatoblasten und Mikrocyten sind sie 2 bis 4 Mikrom. gross, in besonders grossen Hämocyten erreichen sie 10 bis 19 Mikrom., den gesammten Zellraum ausfüllend (Fig. $9 c, d$ ).
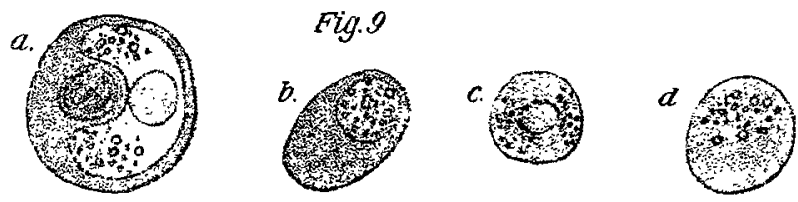

Vergrösserung 1500/1. Ungefärbte inficirte Biutbestandtheile, $b, c, d$ Pseudo vacuolae. a Cystenbildung (?).

Im Blute der Eule sind auch biscuitfürmige, melaninfreie Leukocyten mit solchen Parasiten besetzt. Eine kranke junge Eule Danilewsky's hatte zahlreiche grosse Makrophagen, mit je 1 bis 2 bis 3 Parasiten besetzt, in der Milz und im Knochenmark; im übrigen Körperblut war jeder zweite bis dritte Hämocyt mit ein bis drei Pseudoracuolen besetzt.

Der Zusammenhang dieser runden racuolenartigen Form mit den sichelförmigen Körpern ist durch directe Beobachtung noch nicht festgestellt. Gerade dieser Nachweis wäre wichtig, da die grösseren Pseudovacuolen einen mit eigenartigen Flagellen behafteten, berreglichen Inhalt haben (Polimitus avium Danilewskr). Es ist möglich, dass nur die kleinen Vacuolen, wie bei Lacerta und Emys, mit den sichelfōrmigen Körperchen in Beziehung stehen (Fig. 8a).

Jedenfalls wäre die Entstehung der Polimitusform aus den Psendovacuolen (Fig. 9c,d) eine ganz eigenartige, kaum bei Protozoën, bisher beobachtete. Nur bei Malaria ist ron Lareran, Richard, Golgi, Celli, Marchiafava, Guarnieri u. A. etwas Aehnliches beschrieben (Corps cystiques spheriques Nr. 2 ron Lareran; A. Celli und E. Guarnieri, Taf. III A, Fig. 19, 20), ohne dass der Zusammenhang der geisselbehafteten und Amöboidformen mit den sichelfürmigen Körperchen gefunden ist. Auch die anderweiten Befunde stehen noch ohne Vermittelung neben einander.

Die Deutung dieser Befunde ist wach des Verfassers Ansicht eine ganz andere. Die ron Danilewsky beschriebene Geisselform dürfte kaum als selbstständige zu betrachten sein. Bei rascher Ablïhlung des Präparates (oder Sauerstoffmangel) entsteht nach ein bis zwei Minuten eine Bewegung in der Pseudovacuole; der Hämocyt berstet und es tritt ein kugeliges, 0.006 
bis $0 \cdot 012$ Mikrom. grosses Cytozoon mit 4 bis 10 Geisseln und anhaftenden Plasmaresten aus. (Fig. 10.) Die Geisseln sind 20 bis 30 Mikrom. lang and bewegen sich lebhaft. Ein Kern ist deutlich nachweisbar. An Stelle der Geisseln können auch kürzere, dickere Pseudopodien und ein undulirender Vantel vorhanden sein. Ausstülpungen des Hyaloplasmas, Umbildung zu zwei rereinigten Kugeln, Abstossung der Geisseln und selbstständige Weiterhewegung der letzteren, Zerfall des Körpers zu 10 bis 15 Körnchen unter zuckender Bewegung und mit Zurücklassung eines leeren Sackes - alle diese Erscheinungen sind Zerfallsrorkommnisse, wie sie bei Infusorien und Gregarinen unter ähnlichen misslichen Umständen beobachtet werden.

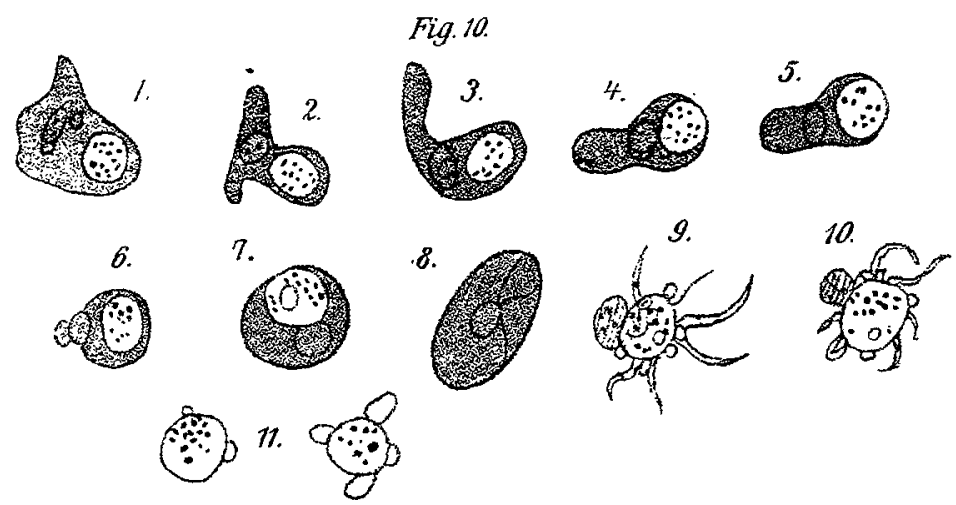

Vergrösserung 1500/1. Bewegliche rothe Blutzellen aus einer SchleiereuIe von Weimar. 8. 9. 10. $11 \mathrm{der}$ ausschlüpfende Polimitus von Danilewsky ( $L_{L}$ P feiffer).

Ob noch weitere von Danilewsky im Vogelblut beobachtete Formgestalten, wie Spirochaeta oder Pseudospirilla (Filaments mobiles im Malariablut nach Laveran, aber von Celli und Guarnieri gar nicht erwähnt) und Trypanosomaformen in den Entwickelungsgang des Polimitus oder der sichelförmigen Kürperchen gehören, ist deshalb gleich ungewiss.

Eine junge Eule mit viel Trypanosoma im Blut erkrankte acut; vier Tage lang fanden sich viel Polimitus im Blute in rerschiedener Grösse und verschwanden mit der Genesung wieder. (Danilewsky.)

Jedenfalls würde durch näheres Studium der Danilewsky'schen neuen Funde auch die Malaria-Aetiologie nur gewinnen können.

\section{Sichelförmige Körper in den Hämocyten bei Malaria.}

Sichelförmige Kürperchen ganz ähnlicher Beschaffenheit, wie siè sueben beschrieben worden sind, kommen innerhalb der rothen Blutscheiben bei gewissell Malariaformen vor. Klinisch und ätiologisch sind. die betreffenden Krankheitsformen noch nicht abgegrenzt. 
Ausser diesen sichelförmigen Körperchen, welche Celli und Guarnieri beschrieben, kommt noch eine amöboide Formengruppe, und zwar viel häufiger, zur Ansicht. Die erste Gruppe kommt an manchen Malariaarten gar nicht ror und ist deshalb sogar ihre Existenz seit der Entdeckung (1881 durch Laveran) jahrelang geläugnet worden.

Möglich, dass es sich um zwei Formen von Malaria, oder um zwei verschiedene Parasiten handelt.

Celli und Guarnieri haben nach ihrer jüngsten Teröffentlichung beide Formenreihen zusammen gesehen, die sichelförmigen Körpercher aber nicht in den typischen Sommerfiebern, sondern nur ausnalimsweise bei der schweren Herbstmalaria und bei Malariacachexie. Tergleichende Untersuchungen aus tropischen Malariaorten, Berücksichtigung des Befundes im Knochenmark, in den NIuskeln, Nieren, Endothelien u. s. w. stehen noch aus. ${ }^{1}$

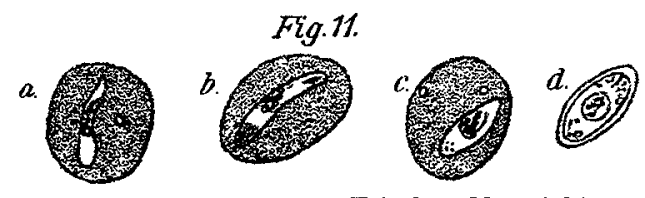

Vergrösserung 1500/1. Frisches Malariablut.

Die vorstehenden Zeichnungen Fig. $11 a-d$ stammen aus dem Fingerblut eines Kaufmannes, der vor $3 / 4$ Jahren zuerst in Triest einen schweren Fieberanfall mit Erbrechen, Ohnmacht gehabt hat. Chinin ist in sehr grossen Mengen und nur mit geringer Lnterbrechung seitdem gebraucht worden. Ein letzter Frostanfall hat Tags vorher, am 30./IX. 1889, zwei Stunden lang stattgehabt. Die Untersuchung ergiebt eine über den Rippenrand hinüberragende Milz, grosse Abmagerung und aschgraue Hautfärbung. Vor dem erneuten Gebrauch ron Chinin ist ein Tröpfchen Blut aus einer Fingerspitze entnommen worden. Das erste Präparat zeigte in drei anscheinend wohl erhaltenen Hämocrten ganz charakteristische sichelförmige Körperchen; in zwei weiteren einen ovalen Fremdling von halber Blutkörperchengrösse. Zwei weitere freie sichelförmige Körperchen wurden noch in anderen Präparaten zwischen den Blutscheiben aufgefunden. Bewegungsvorgänge sind auf dem leider nicht erwärmten Objecttisch nicht zu sehen gewesen. - Durch Zusatz von Methylenblaulösung in Eiweiss an den Rand des Präparates erschien in dem sichelförmigen Körperchen ein die Farbe langsam aufnehmender Kern. - Am ungefärbten Präparat fiel der Fremdling durch seine bläulichweisse Färbung innerhalb der Blutscheibe auf. Eine Trennung des Zooides

1 Vgl. Litteratur Nr. 32 und 47. 
and des Parasiten war nicht zu erkennen. Andere bewegliche oder mit Geisseln behaftete Formen fehlten in den Präparaten. Das Bild der sichelförmigen Körperchen (Fig. 11 $a, b$ ) erinnerte lebhaft an die halbwüchsigen Formen der Haemogregarina cistudinis oder an die den Sarcosporidiencysten des Schafösophagus oder des Schweinemuskels entschlüpfenden sichelförmigen Gestalten.

Wie bei letzteren, nehmen auch hier die beiden Enden den Farbstoff besunders leicht auf. Einer der frei im Blute gefundenen Körper verflachte sich ganz in der Weise, wie zu sehen ist, wenn man Sarcosporidienkeime in Speichel bringt. Leider hat die Untersuchung mit dem gewärmten Uikroskop nicht ausgeführt werden können.

In ganz gleicher Weise, wie Danilewsky die Entwickelung seines Polimitus sanguinis avium in den rothen Blutscheiben, dessen Geburt und lebhafte Geisselbewegung beschreibt, haben Laveran, Richard Marchiafara, Golgi, Osler, Celli und Guarnieri u. A. im Malariablute eine Geisselform gesehen. Es findet hier nach Celli und Guarnieri's jüngster Veröffentlichung ein directer C'ebergang der spindeligen Formen zn den ovalen, von hier zu den runden mit centralen Pigmenthäufchen und von den runden zu den geisselntragenden statt. Nach lebhaften Bewegungen im centralen Protoplasma gehen ron letzterem geisselartige Fortsätze aus, welche energisch oscilliren, um sodann bald stille zu stehen oder sich abzulösen. Verfasser kann nicht auf Grund eigener Beobachtungen angeben, wie weit die bei dem Vogelblut beschriebenen Zerfallsvorkommnisse auch hier sich abspielen.

Das merkwürdigste Phänomen in der Entwickelungsweise des Malariaparasiten, eine Art Knospenbildung der sichelförmigen Keime innerhalb der rothen Blutscheiben, wie es Celli and Guarnieri abbilden, hat Verfasser noch nicht gesehen. Es soll nach deren Beschreibung oft vorvorkommen und ist die directe Abschnürung ron Guarnieri unter dem Deckglas beobachtet worden. An eine zweite nachfolgende Infection desselben Blutkörperchens, wie es bei Emys und Lacerta so oft vorkommt, ist der Abbildung nach nicht zu denken; die Deutung als Kunstproduct bei der Präparation ist nicht ausgeschlossen, da bei Sarcosporidienkeimen mit Essigsäure leicht Plasmatropfen austreten.

Jedenfalls steht diese Infection des Malariablutes mit sichelförmigen Körperchen der Infection des Blutes von Vögeln, von Lacerta und Emys sehr nahe.

Für Emys lutaria liegt ferner durch Danilewsky ein in sich so weit abgeschlossener Cyclus von Entwickelungsformen vor, als für die Gregarinen überhaupt, für Klossia, Eimeria nicht besser erforscht ist. 
Auf diese sichelförmigen Körperchen im Malariablute ist Chinin ohne Einfluss; ihr Vorkommen hängt aber mit Malaria zusammen und hat eine grössere pathogene und diagnostische Bedeutung.

Aehnliche sichelförmige Keime sind im Blute des MLenschen, ausser bei Malaria, noch nicht gesehen worden. Aehnliche sind gefunden in dem Exsudat der Pleurahöhle von Künstler und Pitres ${ }^{1}$. Die Körperchen sind mehr spindelförmig und haben ebenfalls ihren Ursprung in einfachen Cysten. Bei Coccidium perforans und Coccidium oriforme kommen in Leber und Darm an beiden Enden geknöpfte Sporen (die nach Balbiani nochmals in zwei nur einfach geknöpfte zerfallen) vor, die aber frei diselbst nicht gefunden werden. Bei Molluscum contagiosum fehlen sie. Sarcosporidiencysten sind beim Menschen noch nicht gefunden worden. Es wird sich sonach wohl um eine specifische Infection haudeln, deren Zugehörigkeit zu einem oder mehreren klinischen Bildern der Malariaerkrankung noch zu untersuchen ist. Von Th. Smith in Washington ist ein Malariaparasit beim Texasfieber des Rindes aufgefunden worden. ${ }^{1}$

\section{Nachtrag zu Bd. IV dieser Zeitschrift.}

\section{(S. $413 \mathrm{ff}$.)}

Zur Entwickelung der Sarcosporidiencysten.

Die daselbst beschriebene Beweglichkeit der sichelförmigen Keime lässt sich leicht experimentell herstellen, wenn frisches Material mit Speichel warm beobachtet wird. Aus den sichelförmigeu Körpern werden direct amöboide Formen (Fig. 7 b, Bd. IV), welche zur sichelförmigen Gestalt zurückkehren können. $\mathrm{Ob}$ durch diese amöboiden Formen, oder ob durch kleinere Gebilde die Weiterinfection bedingt ist. ist noch nicht aufgeklärt.

An Querschnitten durch Sarcosporidiencysten von Oesophagus, Peritoneum und Pericard des Schafes fällt auf, dass das Centrum der Cysten verödet, während am Rand ein Fortwachsen (bis zu Nussgrösse) statthit. Der Inhalt der centralwärts gelegenen Cysten besteht aus sichelförmigen Körperchen (Fig. 12b); die am Cystenrand gelegenen haben einen Inhalt von runden Zellen (Fig. 12 $a$ ).

Ganz ähnliches Verhalten zeigt sich in den Muskelschläuchen beim Schaf und Schwein, besonders (nicht ausschliesslich) in den Enden des Schlauches.

${ }^{1}$ Siehe L. Pfeiffer, Die Protozoën als Krankheitserreger. Jena. G. Fischer. 1890. 


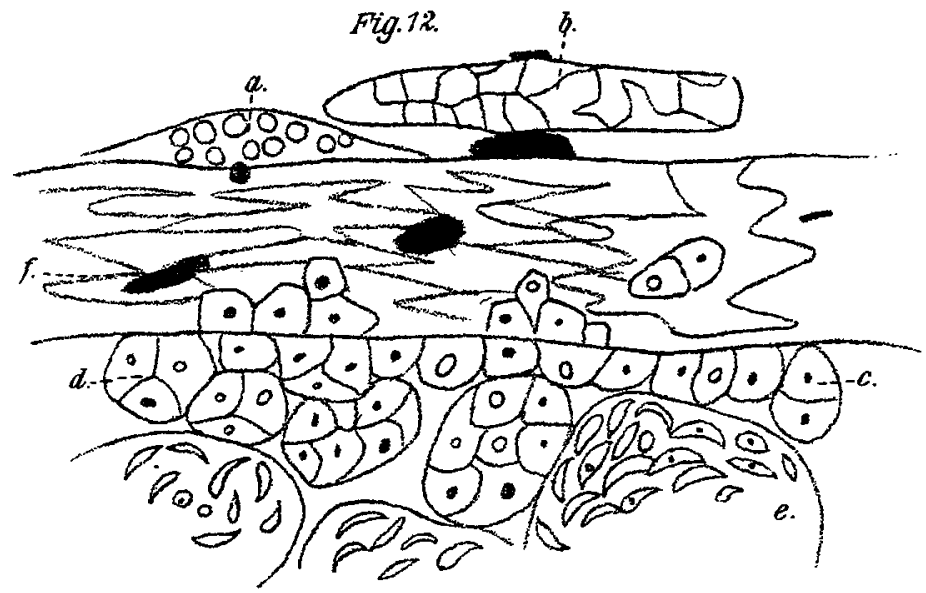

Vergrösserung 1500/1. Querschnitt der Cysten aus einer Sarcosporidie des Schafösophagus, nach $d$ und $e$ hin das Centrum der Cvste gelegen. a Bindegewebe. $b$ Muskelfibrille. Bei $c$ ein- und zweikerniger Sporoblast. $d$ mehr kerniger. $e$ Sporoblast mit sichelförmigem Inhalt. $f$ Muskelkern.

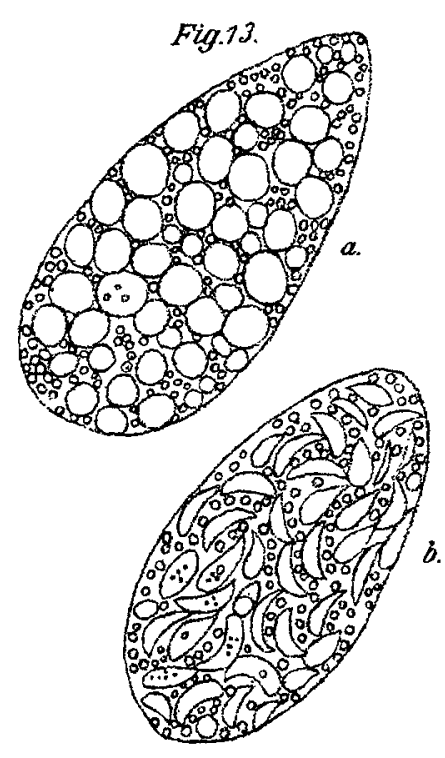

Vergrösserung 1500/1. Theilstücke aus einer Cyste des Schweinemuskels. a Mit runden, $b$ mit weiter entwickelten sichelförmigen Körperchen.

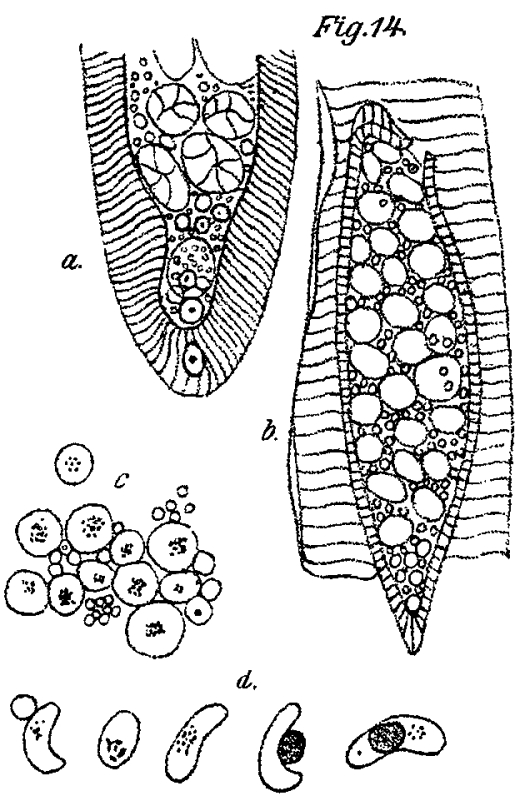

Vergrösserung 1500/1. Muskelfibrillen des Schweines, allerjüngste Formen. a Mit jungen Sporoblasten in den Endstücken, $b$ mit rund. Körperchen in einem einzigen Sporoblasten. $c$ der Inhalt von $b . d$ der Inhalt von $a$. 
In Fig. 12 ist ein Theil eines Schnittes aus der Cystenwand vom Oesophagus des Schafes abgebildet. Bei Fig. $12 b$ ist ein Sporoblast mit sichelförmigen Keimen ausgefüllt; bei Fig. $12 a$ finden sich viele, noch runde Keime mit Kern. Je mehr nach der Peripherie zu, desto mehr nimmt die Zahl der runden Keime ab, und dicht am Rande fanden sich Kammern mit nur je 1 bis 2 Kernen (Fig. 12).

In Fig. 13 und 14 handelt es sich um den gleichen Befund in dem einen Ende eines intrafibrillären Schlauches rom Schwein.

In den Muskelfibrillen der Barbe, welche alljährlich in der Mosel massenhaft an der Psorospermienkrankheit absterben, finden sich die in Fig. 13 abgebildeten Sporoblasten zu grösseren Cysten rereinigt; aber auch in den langen Fibrillen der Bauchmuskeln rosenkranzartig aneinander gereiht, jeder Sporoblast als Individuum mit besonderer C'ystenhaut eingekapselt.

Die Psorospermiencysten, bald solitär (Barbe). bald 10 bis 50, bald 100 und mehr Sporoblasten enthaltend, sind demnach aufzufassen als eine den Cellularschmarotzern eigenthümliche Mehrlingsinfection einer Zelle oder einer Zellgruppe. In Fig. 12 und 13 sind die am Rande der Wirthszelle noch vorhandenen kleineren Schmarotzer nicht durch eine den Gregarinen fremdartige endogene Theilung entstanden, sondern als ein im Wachsthum noch zurückgebliebener Infectionsrest aufzufassen. Ausführlicherer Bericht über diese Intracellularinfection folgt in der zusammenfassenden Arbeit des Verfassers über die Protozoën als Krankheitserreger (G. Fischer, Jena 1890). 


\section{Litteratur-Verzeichniss.}

1. Balbiani, G., Leçons sur les sporozocires. Paris 1884.

2. Derselbe, Les organismes unicellulaires. Journal de micrographie. $1882 \mathrm{ff}$.

3. Baruggi, C., Sulle critiche mosse al Plasmodium malariae. Estratto dal gionale Riforma medica. August 1886.

4. de Bary, A., Vergleichende Morphologie und Biologie der Pilze, Mycetozoën und Bacterien. Leipzig 1884.

5. Blanchard, R., Hématozoaire. Dictionnaire encycl. des sciences méd. pai Lereboulet. 1886.

6. Bütschli, O., Protozoa. Bd. I von H. G. Bronu's Classen und Ordnungen des Thierreichs. I. Sarkodina und Sporozoa. 1880-1882. II. Mastigophora. 1883-1887. III. Infusoria. 1887-1889. Mit 80 Tafeln.

7. Cattaneo, A. und Monti, A., Differentialdiagnose zwischen den zerfallenden rothen Blutkörperchen und den Malariablutkörperchen. Archivio per le scienze mediche. Bd. XII. Nr. 6.

8. Celli, A., Dei protesti citofagi. Estratto della Riforma medica. Maggio 1889.

9. Celli, A., und Guarnieri, E., Sull' Etiologia dell' infezione malarica. Atti della R. accademia med. di Roma. 1888. Vol. IV. Serie II. Deutsch in: Fortschritte der Medicin. 1889. Nr. 14 u. 15. Mit 3 Tafeln.

10. Certes, A., Trypanosoma Balbiani. Bullet. soc. zool. de France. 1882. Vol. VII. p. 7.

11. Cohn, F., Ueber die Aetiologie der Malaria. Schlesische Gesellschaft für. vaterländische Cultur. Juni 1887.

12. Councilman, Neuere Untersuchungen über Laveran's Organismen der Nalaria. Fortschritte der Medicin. 1888. Nr. 12 a. 13.

13. Crookshank, Flagellated protozoa in the blood of diseased and apparently healthy animals. Journ. of the royal microscop. society. 1886. p. 913.

14. Czensinsky, Odessa, Zur Lehre über den Organismus des Malariafiebers. Centralblatt für Bacteriologie. 1888. Nr. 15.

15. Danilewsky, R., Die Hämatozoën der Kaltblüter. Archiv für mikroskop. Anatomie. 1885. Bd. XXIV.

16. Derselbe, Zur Pathologie des Blutes. Biol. Centralbl. 1885. Nr. 17. S. 529.

17. Derselbe, Observations sur une monade (Hexamitus) parasite du sang. Arch. slaves de biologie. 15./II. 1886.

18. Derselbe, Les hématozoaires des lézards. Ebenda. 15./III. 1886. p. 364 bis 396. Mit 2 Tafeln. (Matériaux pour servir à la parasitologie du sang. 15./III. 1886.) 
19. Derselbe, Les cultures capillaires. Arch. slares de biologie. Januar 1886.

20. Derselbe, Zur Erage über die Identität der pathogenen Blutparasiten des Menschen und der Hematozoën der gesunden Thiere. Centralblatt für die medicin. Wissenschaften, 1886 und Archives slaves de biologie. März 18s7.

21. Derselbe, La parasitologie comparée du sang. St. Petersburg bei Karl Ricker. 1889. - I. Nouvelles recherches sur les parasites du sang des oiseaux. Mit 3 Tafeln. - II. Recherches sur les hématozoaires des tortues. MIit 2 Tafeln.

22. Ferrand, Le paludisme à Madagascar. Montpellier 1ssi.

23. Fis $\mathrm{ch}$, Untersuchungen über einige Flagellaten und verwandte Organismen. Zeitschrift für wissenschaftliche Zoologie. 188̄̄. Bd. XLII.

24. Flemming, W., Die Cytozoën. Biologisches Centralblatt. 188̄̄. S. う̌29.

25. Gaule, J, Ueber die Würmchen, welche aus Froschblutkörperchen auswandern. Archiv für Anatomie und Physiologie. 1880. S. 56-6t.

26. Derselbe, Beobachtungen der farblosen Elemente des Froschblutes. Ebenda. 1880. S. 375 . Tafel V.

27. Derselbe, Ebenda. 1880. S. 297. - Centralblatt für medicinische Wissenschaften. 1881. Nr. 31.

28. Derselbe, Ueber die Bedeutung der Cytozoën (Würmchen) zu den Zellkernen. Biologisches Centralblatt. 1886, Bd. VI. S. 345. Mit Tafel V.

29. Gallemaerts, Le microbe de la malaria. Bull. de la soc. de micross. 1888. t. XIV n. II.

30. Golgi, Studi ulteriori sull' infezione malarica. Archivio per le sc. mediche. 1886. Bd. X. Nr.4. - Gazz. degli Ospitali. 1886. Nr. 53. - Deutsch in: Fortschritte der Medicin. 1885. Nr. 24. 1888. Nr. 8. 1899. Nr. 3. S.S1.

31. Derselbe, Ueber den angeblichen Bacillus Malariae von Klebs, Tommasi Crudeli und Schiavuzzi. Beiträge zur pathol. Anatomie von Ziegler und Nauwerck. 1888. Bd. IV. S. 419. - Archivio per le scienze mediche. 1889. Fasc. I.

32. Grassi, B., Intorno ad alcuni protisti endoparasitica (Flagellati, Lobosi, Sporozoi e Ciliati). Atti della Soc. Ital. di scienze naturali. 1882. Vol. XXIV. Auch französisch: Sur quelques protistes endoparasites. Arch. et. de Biol. 1883. t. II. p. 402 und t. III. p. 23.

33. Derselbe, Morfologia e sistematica di alcuui protozoi parasiti. Academia dei Lincei. 8. Jan. 1888. Vol. IV. Fasc. I.

34. Heidenreich, I., Klinische und mikroskopische Čntersuchungen über den Parasiten des Rückfalltyphus. Berlin 1877.

35. Hoffmanu, Tntersuchungen ïher Spaltpilze im menschlichen Blut (bei perniciöser Anämie). Berlin, Hírschwald. 1884. Taf. II. Fig. 14.

36. Klebs, Ed., Allgemeine Pathologie. Jena 1887. Bd.I.

37. Koch, R., Herpetomonas Lewissi im Hamster-, Affen- und Rattenblut. Mittheilungen aus dem Kaiserl. Gesundheitsamt. 1881. Bd. I. Th. 4. S.9.

38. Lancaster, Ray, Undulina ranarum im Frosehblut. Quarterly journal of microsc. science. 1871. t. XI. p. 387-389. - 1882. New series. t. LXXXV. p. 53.

39. Laveran, Nature parasitaire des accidents de l'impaludisme etc. Paris 1881.

40. Derselbe, Traité des fières palustres. 1884. 
41. Derselbe, Des hématozoaires du paludisme. Annales de l'Institut Pasteur. 1887. p. 266. - Juli 1888.

42. Leuckart, Die Parasiten des Menschen. 3. Aufl. 1879-1889.

43. Lewis, Timothy Richard, The microscopic organisms found in the blood of man and animals and their relations to disease. Calcntta 1879. $8^{\circ}$. p. 91 . - Fran. zösische Uebersetzung II. Aufl. 1882. Les mierophytes du sang etc.

44. Derselbe, Flagellated organisms in the blood of healthy rats. Quarterly jounal of microse. science. 1879. p. 109-114.

45. Ebenda. July 1884. Further observations on flagellated organisms in the blood of animals.

46. Marchiafava, Il Plasmodium malariae etc. Rendiconti dei Lincei. Mai 1886. Vol. II. Serie I.

47. Marchiafava e Celli, Sulla infezione malarica. Atti della $R$. Acecdem. Yed. di Roma. 1886. 1887. 1888. - Deutsch in: Fortsehritte der Medicin. 1885. Nr. 11 u. 24.

48. Dieselben, Sul rapporti fra le alterazione del sangue di cane introdotte $e$ t: Bull. della R. Accadem. Med. di Roma. 1886-1887. Vol. III. 1887.

49. Dieselben, Fortschritte der Medicin. 1883. Nr. 18.

50. Dieselben, Blutveränderungen bei Malaria. Atti della R. Academia dei Lincei. 1884.

51. Dieselben, Studi ulteriori sulla infezione malarica. Archivio per le scienze mediche. 1886. Vol. X. Nr.9.

52. Dieselben, Sull intima struttura del Plasmodium malariae. Riforma medica. 1888. Nr. 208 u. 236.

53. Maurel, Recherches microscopiques sur l'étiologie du paludisme. Paris 1887.

54. Metrosanoff, Beiträge zur Kenntniss der Hematozoën. Biolog. Centratblatt.

55. Metschnik off, Zur Lehre von den Malariakrankheiten. Ref. im Centralblatt für Bacteriologie. I. Bd. I. Nr. 21.

56. Mitchell, On the cryptogamous origni of malaria-fecer. Philadelphia 1849. blatt. 1883. Bd. III. S. 35.

57. Osler, W., An adress on the hematozoa of malaria. British med. Journal. 1887. p. 556 .

58. Pfeiffer, L., Das Vorkommen der Marchiafava'schen Plasmodien im Blute von Vaccinirten und Scharlachkranken. Diese Zeitschrift. 1887. Bd. II.

59. Richard, Filaments mobiles de malaria. Compt. rend. 1882 und Revue scientif. 1883. p. 114.

60. Schiavuzzi, -B., Ueber Malaria im Allgemeinen und besonders in Istrien. Internationaler Congress für Hygiene in Wien. 1887. - Rendi conti della R. Academia dei Lincei. Deceember 1886. Mai 1887.

61. von Sehlen, Fortschritte der Medicin. 1884. Bd.

62. Sacharoff, Untersuchungen über deu Parasiten des Malariafiebers. Centralblatt für Bacteriologie. 1889. Nr. 12 u. 13.

63. Sternberg, G. M., The malaria germ of Laveran. The med. Record. 1886. Nr. 18. 
330 L. Pfetffer: Beiträge z. Kenntniss D. Pathogeney Gregarinen.

64. Schelling, Weitere Mittheilungen über die Malaria in Kaiser Wilhelms Land. Deutsche med. Wochenschrift. 1889. Nr. 35 u. 36.

65. Schneider, Aime, Poitiers Contributions à l'étude les gregarines. Arch. de zoologie experimentale et générale. 1882. t. $\mathrm{X}$.

66. Tommasi-Cradeli, Il plasmodium malariae di Marchiafava, Celli e Golgi. Rendi conti della R. Academia dei Lincei. 1886. Bd. II. p. 313.

67. Wallerstein, Ueber Drepanidium ranarum. Ray Jancaster. Dissertation. Binn 1882.

68. Wittich, Spirillen des Blutes vom Hamster. Centralblatt für medicinische Wissenschaften. 1881. Nr. 4.

69. Ziehl, Deutsche med. Wochenschrift. 1882. Nr. $4 \mathrm{~s}$. 